\title{
A mild, expedient, solventless synthesis of bis(indolyl)alkanes using silica sulfuric acid as a reusable catalyst
}

\author{
D. M. Pore ${ }^{a *}$ Uday V. Desai, $^{\mathrm{a}}$ T. S. Thopate, ${ }^{\mathrm{a}}$ and P. P. Wadgaonkar ${ }^{\mathrm{b}}$ \\ ${ }^{a}$ Department of Chemistry, Shivaji University, Kolhapur - 416004, India \\ ${ }^{b}$ Polymer Science \& Engineering Division, National Chemical Laboratory, \\ Pune-411008, India \\ E-mail: p_dattaprasad@rediffmail.com
}

\begin{abstract}
Silica sulfuric acid (SSA) was found to be a mild, efficient and reusable solid acid catalyst in electrophilic substitution reaction of indoles with carbonyl compounds to afford the corresponding bis(indolyl)alkanes in excellent yields under solvent-free conditions.
\end{abstract}

Keywords: Silica sulfuric acid, bis(indolyl)alkanes, aldehydes, ketones, indole

\section{Introduction}

The acid catalyzed reaction of electron rich heterocycles such as pyrroles and indoles with $p$ dimethyl aminobenzaldehyde is known as Ehrlich test[1] and analogues reaction of indoles with other aldehydes and ketones affords corresponding bis(indolyl)alkanes, a useful class of organic compounds. A few bioactive members of this class like Vibrindole-A and others have also been isolated from natural sources[2] and are found to be pharmaceutically important.[3] Especially, bis(indolyl)methane is known to promote estrogen metabolism in both women and men and is expected to have an application in the prevention of breast cancer.[4] Due to such pharmaceutical importance the synthesis of bis(indolyl)methane and its derivatives is receiving considerable attention these days.[5]

The electrophilic substitution reaction of indoles with carbonyl compounds to produce bis(indolyl)alkane is an acid catalyzed reaction and both protic[6] as well as Lewis acids[7] are known to promote this reaction. However, many Lewis acids are prone to undergo decomposition in the presence of nitrogen containing reactants and this necessitates the use of excess and sometimes stoichiometric amount of Lewis acid catalyst.[8] To overcome such problems, recently Montmorillonite K-10,[9,2a] triphenylphosphonium perchlorate, lithium perchlorate,[10,11] iodine,[12] zeolites,[13] triflate of lanthanides[14] and cyanuric chloride[15] have been reported to catalyze this reaction. However, many of these reagents are expensive, 
$[10,11,14]$ need longer reaction time and are not environmental friendly which warrants the development of a new, practical, economical and environmental friendly protocol for the synthesis of bis(indolyl)methanes.

The use of solid acid catalysts has received considerable attention in organic synthesis due to their environmental compatibility, ease of handling, non- toxic nature and above all their reusability[16] and the development of solventless protocol has an added advantage in green context. Recently, silica sulfuric acid (SSA) has been used as a solid acid catalyst in many organic transformations [17] including the chemoselective tetrahydropyranylation of alcohols[18] as well as chemoselective dithioacetalization of aldehydes[19] reported by us and most recently in Dakin -West reaction.[20] Herein we wish to report the use of SSA as a reusable solid acid catalyst for the synthesis of bis(indolyl)methanes.

\section{Results and Discussion}

As a case study when a mixture of $p$-methoxybenzaldehyde $(2 \mathrm{mmol})$, indole or indole derivative (4 mmol) and SSA ( $100 \mathrm{mg}, 0.26 \mathrm{mmol}$ ) was stirred at room temperature, corresponding bis (indolyl)methane was formed (TLC) in a short reaction time (Scheme 1).

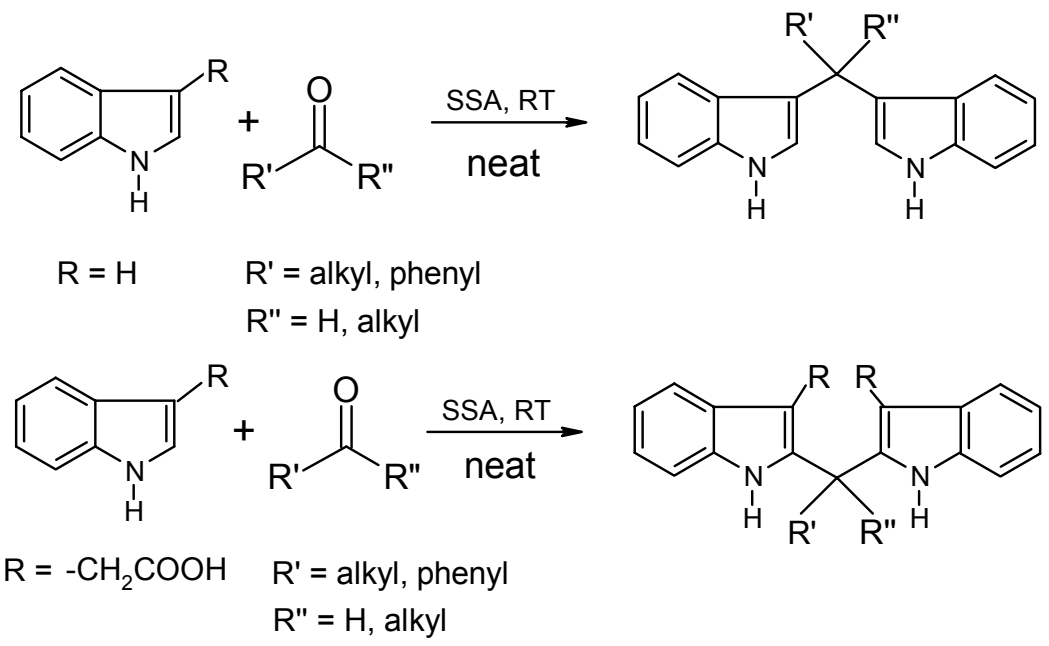

\section{Scheme 1}

To prove the generality of the protocol, the reaction was then extended towards a variety of aldehydes as well as ketones with indole as well as indole- 3- acetic acid and the results are summarized in Table-1. Though the reactions of indole with various aldehydes were fast, the reaction with ketones took relatively longer time (Entry 7, Table 1). As expected the electron rich aldehydes reacted faster than the aldehydes with electron withdrawing group (Entry 2-5, Table 1). Instead of indole, when the reaction was extended towards indole-3-acetic acid, the electrophilic substitution occurred at C-2 in acceptable yields (Entry 8, Table 1). 
Table 1. Silica Sulfuric acid catalyzed synthesis of bis(indolyl) methanes

\begin{tabular}{|c|c|c|c|c|c|c|}
\hline Entry & Indole & $\begin{array}{l}\text { Aldehyde/ } \\
\text { Ketone }\end{array}$ & Product & $\begin{array}{l}\text { Time } \\
(\min )\end{array}$ & $\begin{array}{l}\text { Yield } \\
(\%)^{\mathrm{a}, \mathrm{b}}\end{array}$ & $\begin{array}{c}\mathrm{mp}{ }^{\circ} \mathrm{C} \\
\text { Obs (Lit) }\end{array}$ \\
\hline 1 & & & & 40 & 92 & $\begin{array}{l}125-127 \\
\left(125-126^{12 b}\right)\end{array}$ \\
\hline 2 & & & & 10 & 89 & $\begin{array}{l}97-99 \\
\left(95-97^{11}\right)\end{array}$ \\
\hline 3 & & & & 15 & 94 & $\begin{array}{l}178-181 \\
\left(179-181^{13}\right)\end{array}$ \\
\hline 4 & & & & 10 & 95 & $\begin{array}{l}196-197 \\
\left(198-200^{11}\right)\end{array}$ \\
\hline 5 & & & & 30 & 90 & $\begin{array}{l}120-122 \\
\left(104-105^{13}\right)\end{array}$ \\
\hline 6 & & & & 10 & 93 & $\begin{array}{l}113-115 \\
\left(113-115^{13}\right)\end{array}$ \\
\hline 7 & & & & $2.5^{\mathrm{c}}$ & 67 & $\begin{array}{l}116-118 \\
\left(118-120^{11}\right)\end{array}$ \\
\hline 8 & & & & $6^{\mathrm{c}}$ & 82 & $\begin{array}{l}123-125 \\
\left(125-130^{15}\right)\end{array}$ \\
\hline
\end{tabular}

${ }^{\mathrm{a}}$ Yields refer to pure, isolated products; ${ }^{\mathrm{b}}$ All the products gave satisfactory spectroscopic ( IR, ${ }^{1} \mathrm{H}$, and ${ }^{13} \mathrm{C}$ NMR) analysis; ${ }^{\mathrm{c}}$ Time in hours. 
To check the reusability of catalyst, when the reaction of indole with carbonyl compound was over the product formed was extracted with chloroform and the catalyst was filtered. It was washed with chloroform repeatedly, dried and reused for the reaction of indole with the same or different aldehydes. It was found that catalyst can be recycled for atleast three cycles without any change in activity.

In conclusion, silica sulfuric acid was found to be a mild, efficient and reusable solid acid catalyst for the reactions of indoles with aldehydes and ketones to furnish bis(indolyl)alkanes, under solvent-free conditions.

\section{Experimental Section}

General procedure. A mixture of aldehyde $(2 \mathrm{mmol})$, indole / indole-3-acetic acid (4 mmol) and SSA (100 mg, $0.26 \mathrm{mmol}$ ) was stirred at room temperature for specified period. On completion of reaction (TLC) chloroform $(20 \mathrm{~mL})$ was added and the reaction mixture filtered. The catalyst was washed with chloroform $(2 \times 10 \mathrm{~mL})$. Removal of solvent from combined filtrate gave the residue, which was filtered through a column of silica gel to afford pure bis(indolyl)alkane which was characterized by spectral methods. The spectral data of some of the bis(indolyl)methanes is summarized below.

\section{Spectroscopic data}

3,3'-Bis(indolyl) phenylmethane (Entry 1). Mp 125-127 ${ }^{\circ} \mathrm{C}$; (Lit.[12b] 125-126 ${ }^{\circ} \mathrm{C}$ ) IR (KBr): 3478, 3019, 1601, 1522, 1456, 1419, 1215, 1093, 1017, 757, $669 \mathrm{~cm}^{-1}$; PMR $\left(\mathrm{CDCl}_{3}\right)$ : $\delta_{\mathrm{H}} 5.89(1 \mathrm{H}, \mathrm{s}), 6.67(2 \mathrm{H}, \mathrm{s}), 7.09-7.58(13 \mathrm{H}, \mathrm{m}), 7.94(2 \mathrm{H}, \mathrm{bs}, \mathrm{NH}) ; \mathrm{CMR}\left(\mathrm{CDCl}_{3}\right): \delta_{\mathrm{C}} 143.5$ $136.6,128.5,126.8123 .5,122,119.8,119.4,119.1,111,40$.

3,3'-Bis(indolyl)-4-methylphenylmethane (Entry 2). Mp 97-99 ${ }^{\circ} \mathrm{C}$; (Lit.[11] 95-97 ${ }^{\circ} \mathrm{C}$ ) IR (KBr): 3480, 3020, 1602, 1512, 1456, 1417, 1215, 1091, 1021,759, $669 \mathrm{~cm}^{-1}$; PMR $\left(\mathrm{CDCl}_{3}\right)$ : $\delta_{\mathrm{H}} 2.31(3 \mathrm{H}, \mathrm{s}), 5.84(1 \mathrm{H}, \mathrm{s}), 6.64(2 \mathrm{H}, \mathrm{s}), 6.85-7.40(12 \mathrm{H}, \mathrm{m}), 7.94(2 \mathrm{H}, \mathrm{bs}, \mathrm{NH}) ; \mathrm{CMR}\left(\mathrm{CDCl}_{3}\right)$ : $\delta_{\mathrm{C}} 136.7,128.9,127.1,123.5,121.9119 .9,119.2,110 ., 55.9,39.8$.

3,3'-Bis(indolyl)-4-methoxyphenylmethane (Entry 3). Mp 178-181 ${ }^{\circ} \mathrm{C}$; (Lit.[13] 179-181 ${ }^{\circ} \mathrm{C}$ ) IR (KBr): 3480, 3019, 2838, 1610, 1509, 1455, 1456, 1417, 1336, 1216, 1091, 1033, $759 \mathrm{~cm}^{-1}$; PMR $\left(\mathrm{CDCl}_{3}\right): \delta_{\mathrm{H}} 3.77(3 \mathrm{H}, \mathrm{s}), 5.84(1 \mathrm{H}, \mathrm{s}), 6.64(2 \mathrm{H}, \mathrm{d}), 6.83(2 \mathrm{H} \mathrm{d}), 7.03(2 \mathrm{H} \mathrm{t}), 7.2(2 \mathrm{H}, \mathrm{t}) ; 7.26-$ 7.40(6H, m), 7.89(2H, bs, NH); CMR $\left(\mathrm{CDCl}_{3}\right): \delta_{\mathrm{C}} 136.7,136.2,127.1,123.5,121.9,120$, $119.9,119.4,119.2,113.6,110.9,55.2,38.6$.

3,3'-Bis(indolyl)-3,4-dimethoxyphenylmethane (Entry 4). Mp 196-197 ${ }^{\circ} \mathrm{C}$;(Lit.[11] 198-200 $\left.{ }^{\circ} \mathrm{C}\right)$ IR (KBr): 3480, 3020, 1604, 1512, 1456, 1418, 1336, 1216, 1091, 1033, $759 \mathrm{~cm}^{-1}$; PMR $\left(\mathrm{CDCl}_{3}\right): \delta_{\mathrm{H}} 3.76(3 \mathrm{H}, \mathrm{s}), 3.85(3 \mathrm{H}, \mathrm{s}), 5.83(1 \mathrm{H}, \mathrm{s}), 6.65(2 \mathrm{H}, \mathrm{d}), 6.78(2 \mathrm{H}, \mathrm{d}), 7.0(3 \mathrm{H}, \mathrm{t})$, 7.17(2H, t), 7.29-7.43(4H, m), 7.91(2H, bs, NH); $\mathrm{CMR}\left(\mathrm{CDCl}_{3}\right): \delta_{\mathrm{C}} 147.4,136.7,127.1,123.5$, $121.9,119.8,119.2,112.3,111,55.8,39.8$.

3,3'-Bis(indolyl)-4-chlorophenylmethane (Entry 5). Mp120-122 ${ }^{\circ} \mathrm{C}$; (Lit.[13] 104-105 ${ }^{\circ} \mathrm{C}$ ). IR (KBr): 3478, 3020, 2927, 1600, 1523, 1456, 1417, 1216, 1091, 1015, $759670 \mathrm{~cm}^{-1}$; PMR 
$\left(\mathrm{CDCl}_{3}\right): \delta_{\mathrm{H}} 5.88(1 \mathrm{H}, \mathrm{s}), 6.63(2 \mathrm{H}, \mathrm{brs}), 7.00-7.70(12 \mathrm{H}, \mathrm{m}), 7.92(2 \mathrm{H}, \mathrm{bs}, \mathrm{NH}) ; \mathrm{CMR}\left(\mathrm{CDCl}_{3}\right)$ : $\delta_{\mathrm{C}} 136.7,130.1,128.1,126.8,123.5,122,119.8,119.4,119.3,111,39.8$.

3,3'-Bisindolyl(2-furfuryl)methane (Entry 6). Mp113-115 ${ }^{\circ} \mathrm{C}$; (Lit.[13] 113-115 ${ }^{\circ} \mathrm{C}$ ) IR (KBr): 3477, 3019, 2399, 1600, 1456, 1419, 1216, 1093, 1021, 757, $670 \mathrm{~cm}^{-1} ; \mathrm{PMR}\left(\mathrm{CDCl}_{3}\right): \delta_{\mathrm{H}} 5.94$ $(1 \mathrm{H}, \mathrm{s}), 6.06(1 \mathrm{H}, \mathrm{d}), 6.30(1 \mathrm{H}, \mathrm{d}), 6.87(1 \mathrm{H}, \mathrm{d}), 7.08(2 \mathrm{H}, \mathrm{t}), 7.17(2 \mathrm{H}, \mathrm{t}), 7.29-7.48(5 \mathrm{H}, \mathrm{m})$, $7.95(2 \mathrm{H}, \mathrm{bs}, \mathrm{NH})$.

1,1-(3,3'-Bisindolyl)cyclohexane (Entry 7). Mp116-118 ${ }^{\circ} \mathrm{C}$; (Lit. [14] 118-120 $\left.{ }^{\circ} \mathrm{C}\right)$; IR (KBr): 3478, 3020, 2935, 1603, 1522, 1421, 1335, 1216, 1099, 1017, 758, $669 \mathrm{~cm}^{-1}$; PMR $\left(\mathrm{CDCl}_{3}\right)$ : $\delta_{\mathrm{H}} 1.56(6 \mathrm{H}, \mathrm{m}), 2.48(4 \mathrm{H}, \mathrm{m}), 6.81(2 \mathrm{H}, \mathrm{t}), 7.03(4 \mathrm{H}, \mathrm{m}), 7.20(4 \mathrm{H}, \mathrm{m}), 7.50(2 \mathrm{H}, \mathrm{d}), 7.87(2 \mathrm{H}, \mathrm{bs}$, $\mathrm{NH})$.

\section{Acknowledgements}

One of the authors (UVD) wishes to thank UGC, New Delhi for financial assistance [F.30-72/2004(SR)] and T. S. Thopate thanks UGC authorities for the award of Teacher Fellowship under Faculty Improvement Programme.

\section{References}

1. (a) Morgan, L.; Schunior, R. J. Org. Chem. 1962, 27, 3696. (b) Dolphin, D. J. Heterocycl. Chem. 1970, 7, 275.

2. (a) Chakrabarty, M.; Basak, R.; Harigaya, Y.; Ghosh, N. Tetrahedron Lett. 2002, 43, 4075. (b) Bell, R.; Carmeli, S.; Sar, N. J. Nat. Prod. 1994, 57, 1587.

3. Sundberg, R. J. Academic Press: New York, 1970.

4. Karthik, M.; Tripathi, A. K.; Gupta, N. M.; Palanichamy, M.; Murugeson, V. Cat. Commun. 2004, 5, 371 and references cited therein.

5. Gribble, G. W. J. Chem. Soc., Perkin Trans 1 2000, 1045.

6. (a) Roomi, M.; MacDonald, S. Can. J. Chem. 1970, 48, 139-143. (b) Gregorovich, B.; Liang, K.; Clugston, D.; MacDonald, S. Can. J. Chem. 1968, 46, 3291.

7. (a) Banerji, J.; Chatterji, A.; Manna, S.; Pascard, C.; Prange, T.; Shoolery, J. Heterocycles 1981, 15, 325. J. Chem. Soc., Perkin Trans. I 1980, 553. (b) Babu, G.; Sridhar, N.; Perumal, P. T. Synth. Commun. 2000, 30, 1609.

8. Kobayashi, S.; Araki, M.; Yasuda, M. Tetrahedron Lett. 1995, 36, 5773.

9. Maiti, M.; Bhattacharyya, P. J. Chem. Res. 1997, 424.

10. Nagarajan, R.; Perumal, P. T. Synth. Commun. 2002, 32, 105.

11. Yadav, Jhillu. S.; Subba Reddy, B. V.; Murthy Ch. V. S. R.; Mahesh Kumar.; Madan Ch. Synthesis 2001, 783.

12. a) Bandgar, B. P.; Shaikh, A. K. Tetrahedron Lett. 2003, 44, 1959. b) Bandgar, B. P.; Bettigeri, S. A.; Joshi, N. S. Monatshefte fur Chemie 2004, 135, 1265. 
13. Reddy, A. V.; Ravinder, K.; Nagarajan Reddy, V. L.; Gound, T. V.; Ravikanth, V.; Venkateswarlu, Y. Synth. Commun. 2003, 33, 3687.

14. (a) Chen, D.; Yu, L.; Wang, P. G. Tetrahedron Lett. 1996, 37, 4467. (b) Ji, P. J.; Zhou, M. F.; Qu, D. G.; Wang, S. V.; Loh, T. P. Synlett 2003, 2077.

15. Sharma, G. V. M.; Reddy, J. J.; Lakshmi, P. S.; Krishna, P. R. Tetrahedron Lett. 2004, 45, 7729.

16. Shaabani, A.; Soleimani, K.; Bazgir, A. Synth. Commun. 2004, 34, 3303.

17. (a) Zolfigol, M. A. Tetrahedron 2001, 57, 9509. (b) Zolfigol, M. A; Chehardol, G.; Mallakpour, S. E. Synth. Commun. 2003, 33, 833. (c) Zolfigol M. A.; Bamoniri, A Synlett. 2002, 1621. (d) Zolfigol, M. A.; Mirjalili, B. F; Bamoniri, A.; Karimi Zarchi, M. A.; Zarei, A.; Khazdooz, L.; Noei, J Synlett 2002, 1621 and references cited therein.(e) Zolfigol, M. A.; salehi, P.; Shiri, M. Phosphorus, Sulphur and Silicon 2004, 179, 2273. (f) Li, J. -T; Dai, H. -G; Xu, W. -Z; Li, T. -S. J. Chem. Res.(S), 2006, (1), 41.

18. Pore, D. M.; Desai, U. V.; Mane, R. B.; Wadgaonkar, P. P. Synth Commun. 2004, 34, 2135.

19. Pore, D. M.; Desai U. V.; Mane R. B.; Wadgaonkar P. P. Indian J. Chem. Sec. B 2006, 45B, 1291.

20. Khodaei, M. M.; Khosropour, A. R.; Fattahpour, P. Tetrahedron Lett, 2005, 46, 2105. 